\title{
Biodegradable and Multifunctional Polymer Micro-Tubes for Targeting Photothermal Therapy
}

\author{
Xin Wang ${ }^{1}$, Guoping Yu ${ }^{1, *}$, Xiyu Han ${ }^{2,3}$, Hua Zhang ${ }^{4}$, Jing Ren ${ }^{1}$, Xia Wu ${ }^{1}$ and Yanfeng Qu ${ }^{1}$ \\ 1 Food Science College, Northeast Agricultural University, 59 GongBin Road, XiangFang District, \\ Harbin 150030, China; E-Mails: neauwangxin@gmail.com (X.W.); renjing1979@126.com (J.R.); \\ wuxiatt@126.com (X.W.); Yanfengqu@gmail.com (Y.Q.) \\ 2 School of Clinical Medical, Heilongjiang University of Chinese Medicine, Harbin 150040, China; \\ E-Mail: xiyuhan@gmail.com \\ 3 Heilongjiang International Travel Healthcare Center, 9 GanShui Road, Harbin 150036, China \\ 4 School of Food Science and Engineering, Harbin Institute of Technology, 73 HuangHe Road, \\ NanGang District, Harbin 150090, China; E-Mail: zhhua@hit.edu.cn \\ * Author to whom correspondence should be addressed; E-Mail: yuguoping@neau.edu.cn; \\ Tel./Fax: +86-451-5519-0637.
}

Received: 2 May 2014 / in revised form: 30 May 2014 / Accepted: 24 June 2014 /

Published: 2 July 2014

\begin{abstract}
We describe an innovative form of polymer micro-tubes with diverse functions including biodegradation, magnetic manipulation, and photothermal effect that employs and activates photothermal therapy to target cancer cells. The micro-tube comprised soybean protein isolate, poly-L-glutamic acid, magnetite nanoparticles, plus gold nanoparticles. Through electrostatic force, these components, with opposite charges, formed pairs of layers in the pores of the template, various bilayers of soybean protein isolate and poly-L-glutamic acid served as the biodegradable building wall to each micro-tube. The layers of magnetite nanoparticle functionalized micro-tubes enabled the micro-tube manipulate to target the cancer cells by using an external magnetic field. The photo-thermal effect of the layer of gold nanoparticles on the outer surface of the micro-tubes, when under irradiation and when brought about by the near infrared radiation, elevated each sample's temperature. In addition, and when under the exposure of the near infrared radiation, the elevated temperature of the suspension of the micro-tubes, likewise with a concentration of $0.2 \mathrm{mg} / \mathrm{mL}$, and similarly with a power of $2 \mathrm{~W}$ and as well maintained for $10 \mathrm{~min}$, elevated the temperature of the suspension beyond $42{ }^{\circ} \mathrm{C}$. Such temperatures induced apoptosis of target cancer cells through the effect of photothermal therapy. The
\end{abstract}


findings assert that structured micro-tubes have a promising application as a photothermal agent. From this assertion, the implications are that this multifunctional agent will significantly improve the methodology for cancer diagnosis and therapy.

Keywords: micro-tube; soybean protein isolate; magnetic manipulation; photothermal therapy

\section{Introduction}

Photothermal therapy offers a promising treatment for premalignant and early-stage cancer due to its minimal and diffusive nature and deep tissue penetration [1,2]. The combination of near infrared radiation (NIR), and a corresponding photothermal agent, was used to perform physical therapy to destroy cancer cells [3]. When treated by NIR, the photothermal agent (PTA) converted the light energy to a heat source and then rapidly increased the local temperature. The elevated temperature in the vicinity of the PDA then triggered apoptosis within the cancer cell [4].

Among the diverse range of photothermal agents, gold nanoparticles (Au NPs) are used in biological and medical applications because of their beneficial biocompatibility and optical properties [5]. By controlling the size and geometry of the Au NPs, the wavelength of plasmon resonance of the Au NPs were tuned to the region of the NIR with minimal absorption and effective penetration when related to human tissue. However, in relation to photothermal therapy, a requirement is that the PTAs are located. However, in spite of this requirement in relation to photothermal therapy, and before conducting the therapy, another requirement is that the PTAs are located to the target cancer cells.

In addition, to avoid the adverse effect on normal cells, degradation of the PTAs was required after the treatment of the NIR. Likewise, the inherent drawback of the PTAs limited the application of photothermal therapy. Hence, the photothermal therapy offer challenges to develop degradable PTAs with a controllable location to human tissue. To address this problem the experiment employed a form of polymer micro-tubes with diverse functions including biodegradation, magnetic manipulation, and photothermal effect; the experiment then employed and activated photothermal effect to target cancer cells.

With regard to photothermal agents, most past research efforts, found within the applicable research literature, have focused on spherical particles, such as porphyrin bilayer cerasomes [6], together with micro-capsules [7]. In this experiment, the tubular structure of photothermal agents provided an opportunity to develop multifunctional micro-tubes by the application of inner and outer surfaces [8]. In conjunction with a micro-porous template, the construction of the polymer micro-tube is based on the electrostatic Layer-by-Layer (LbL) technique [9]. To achieve this outcome, two compositions of micro-tube soybean protein isolate (SPI) and poly-L-glutamic acid (PGA) were alternately deposited on the pores of the template through electrostatic interaction to form biodegradable building walls on each micro-tube [10].

The $\mathrm{Fe}_{3} \mathrm{O}_{4}$ NPs and the $\mathrm{Au}$ NPs were correspondingly assembled to the inner and outer surfaces of the micro-tubes. By applying the external magnetic field, the micro-tubes containing the $\mathrm{Fe}_{3} \mathrm{O}_{4} \mathrm{NPs}$ enabled magnetic manipulation, which were then directed to the targeted tissue location [11]. To enhance the performance of photothermal therapy, the assembly process causes the aggregation of $\mathrm{Au}$ NPs on the microtube, which allows for strong absorption in the NIR region. In this case, such 
multifunctional polymer micro-tubes, by remote control, provide a method to target cancer cell photo-thermal therapy. In addition, the findings also offer new candidates promising treatment for premalignant and early-stage cancer by offering photothermal therapy in this new practical way.

\section{Results and Discussion}

\subsection{Fabrication of the Multifunctional Polymer Micro-Tubes}

Depicted in Figure 1, and summarized in three steps, is the process of fabricating the multifunctional polymer micro-tubes that include: (1) The preparation of the polymer micro-tubes; (2) The releasing of the micro-tubes from the template; (3) The assembly of Au NPs on the outer surface of the micro-tube.

The $\mathrm{Au}$ NPs, with a size of $2-5 \mathrm{~nm}$ and the $\mathrm{Fe}_{3} \mathrm{O}_{4} \mathrm{NPs}$, with average diameter of $15 \mathrm{~nm}$, were synthesized according to the reported methods $[12,13]$ The preparation of the micro-tubes, with SPI and PGA, were based on the integration of polymers into the porous template. The positive charged PGA and the negative charged SPI, as a thin film, and one bilayer of (PGA/SPI), were alternately assembled into the pores of the template by immersing the template into each solution. To form the durable polymer building walls of the micro-tubes with PGA on the inner and outer surfaces of the micro-tubes, 14.5 bilayers of (PGA/SPI) thin film was placed into the template pores. Noticing that number of layers are counted by preparation procedure, the successful assembly of the components on the template has been reported [11]. To fabricate the magnetic micro-tubes, the stabilized citrate was then integrated into the pores of the template. The unwanted materials were then removed by polishing the surfaces of the template using a wet cotton swab.

Figure 1. Schematic illustration of the fabrication of $\mathrm{Au} \mathrm{NPs}-(\mathrm{PGA} / \mathrm{SPI})_{14.5}-\mathrm{Fe}_{3} \mathrm{O}_{4} \mathrm{NPs}$ micro-tubes. The blue porous pie represents the template for deposition of components. The brown dots and the colorful bands inside the micro-tube represent magnetic nanoparticles and (poly-L-glutamic acid) (PGA)/soybean protein isolate (SPI) multilayers, and the black dots on the outer surfaces of the micro-tubes represent the Au NPs.

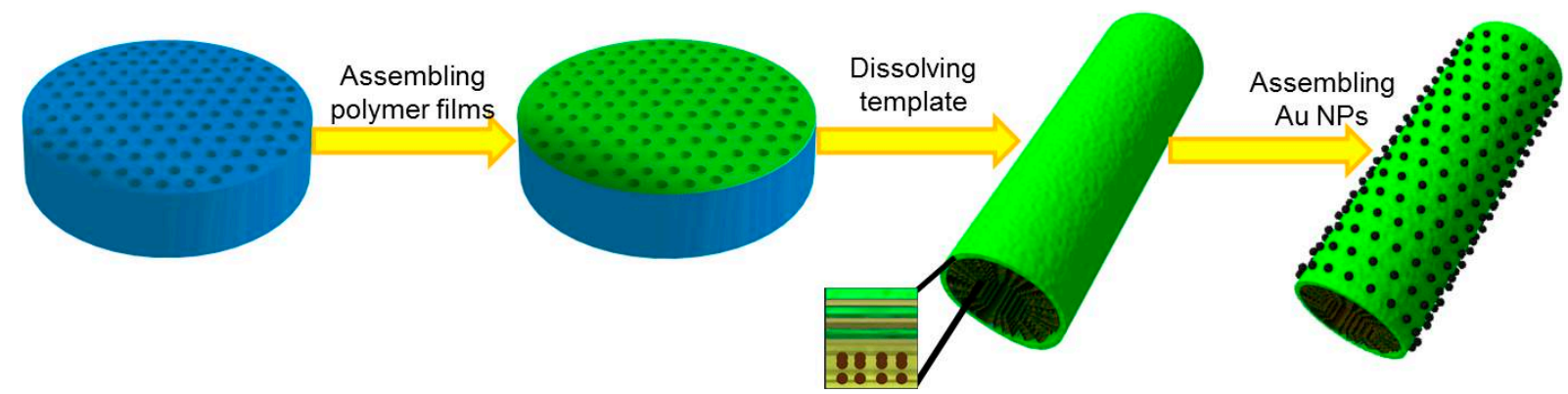

The micro-tubes, with a positive charged PGA on the outer surface, were then released by resolving the template with $\mathrm{CH}_{2} \mathrm{Cl}_{2}$ and diffusing, independently, the template in both ethanol and water. This procedure was followed by the absorption of the negatively charged Au NPs onto the PGA layer by electrostatic interaction. The resulting $\mathrm{Au}$ NPs-(PGA/SPI $)_{14.5}-\mathrm{Fe}_{3} \mathrm{O}_{4}$ NPs micro-tubes were then collected by the external magnetic field. 


\subsection{Characterization of the Gold Nanoshelled Micro-Tubes}

The scanning electronic microscope (SEM) images of a single Au NPs-(PGA/SPI) ${ }_{14.5}-\mathrm{Fe}_{3} \mathrm{O}_{4}$ NPs micro-tube are illustrated in Figure 2a. This micro-tube displays a typical cylindrical geometry with length of $10 \mu \mathrm{m}$; the length of this micro-tube is in accordance with the thickness of the template. The diameter of the micro-tube was approximately $5 \mu \mathrm{m}$, which is in accordance with the pore diameter of the template used.

The corresponding energy-dispersive X-ray (EDX) spectrum verified the presence of both $\mathrm{Au}$ (Figure $2 \mathrm{~b}$ ) and $\mathrm{Fe}$ (Figure 2c). This outcome signified the successful assembly of the $\mathrm{Fe}_{3} \mathrm{O}_{4} \mathrm{NPs}_{\mathrm{s}}$ with a dimensional measurement of 10-30 nm (Figure 2e) and the Au NPs with a dimensional measurement of 2-5 nm (Figure 2f). The following enlarged SEM image shows the high coverage of the Au NPs over the micro-tube surface.

Figure 2. The characterization of the micro-tube. (a) SEM image of a single micro-tube; (b) The corresponding energy-dispersive X-ray (EDX) mapping images of (a) for $\mathrm{Au}$ distribution; (c) The corresponding EDX mapping images of (a) for $\mathrm{Fe}$ distribution; (d) Enlarged SEM image of the micro-tube; (e) TEM images of $\mathrm{Fe}_{3} \mathrm{O}_{4} \mathrm{NPs}$; (f) TEM images of Au NPs.

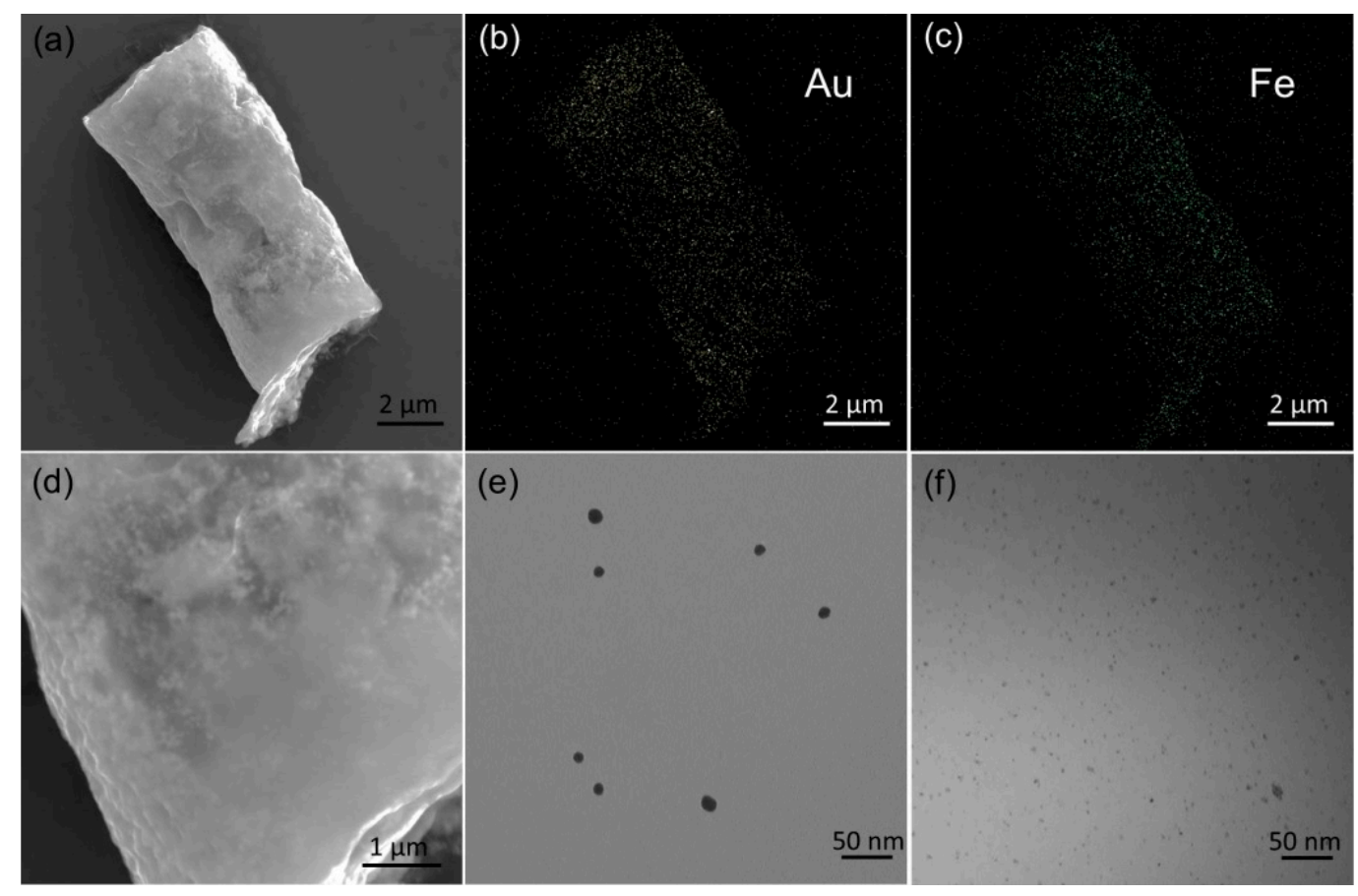

\subsection{Magnetic Manipulation of the Micro-Tubes}

The outer and inner surfaces of the Au NPs-(PGA/SPI $)_{14.5}-\mathrm{Fe}_{3} \mathrm{O}_{4}$ NPs micro-tubes were constructed to have two functions. To conduct the photothermal effect, the first function united the Au NPs on the outer surface of the micro-tubes. Then, to perform the magnetic manipulation of the micro-tubes, the second function assembled the $\mathrm{Fe}_{3} \mathrm{O}_{4}$ NPs on the inner surface of the micro-tubes. Consequently, under the irradiation of the NIR, the Au NPs-(PGA/SPI $)_{14.5}-\mathrm{Fe}_{3} \mathrm{O}_{4}$ NPs micro-tubes elevated the temperature through the photo-thermal effect of the Au NPs. In this situation, the micro-tubes were manipulated by 
using an external magnet (Figure 3a). The micro-tubes were rapidly attracted to the magnet and the black suspension of the micro-tubes became almost colorless. In addition, in the absence of the external magnetic field, the attracted micro-tubes were re-dispersed in a medium. In this way, the micro-tubes were guided to the target cancer cells by using the external magnetic field. It should be noted that the assembly of the $\mathrm{Fe}_{3} \mathrm{O}_{4}$ NPs to the microtube allows for the effective external magnetic manipulation in the compare to bare $\mathrm{Fe}_{3} \mathrm{O}_{4} \mathrm{NPs}$, because of the weak magnetism of a single ultrasmall $\mathrm{Fe}_{3} \mathrm{O}_{4} \mathrm{NP}$.

As shown in Figure $3 \mathrm{~b}-\mathrm{d}$, the locomotion of a micro-tube in the cell culture occurred over a period of $8 \mathrm{~s}$ at 4-s intervals; following this process the micro-tube floated on the surface of the cell culture (Figure 3b). When under the exposure of external magnetic field, the cell culture was located to the HeLa cells (Figure 3b,c); the magnetic micro-tubes were easily manipulated and the magnetic property of the micro-tubes was maintained for more than one month at $4{ }^{\circ} \mathrm{C}$. More importantly, such protocol of microtube also allows for the targeted movement by other physical stimuli (including the light, heat and even osmotic pressure) or the affinity molecules like antibody, aptamer, and target peptide.

Figure 3. The magnetic manipulation of the $\mathrm{Au}$ NPs-(PGA/SPI ${ }_{14.5}-\mathrm{Fe}_{3} \mathrm{O}_{4}$ NPs micro-tubes. (a) This photograph showing the aggregation of the Au NPs-(PGA/SPI $)_{14.5}-\mathrm{Fe}_{3} \mathrm{O}_{4} \mathrm{NPs}$ micro-tubes under the external magnetic; (b-d) Time-lapse images illustrating the movement of a single micro-tube to the HeLa cells, the blue line shows the trajectory of the locomotion of the micro-tube to the HeLa cells under the external magnetic field. Scale bar $=20 \mu \mathrm{m}$.

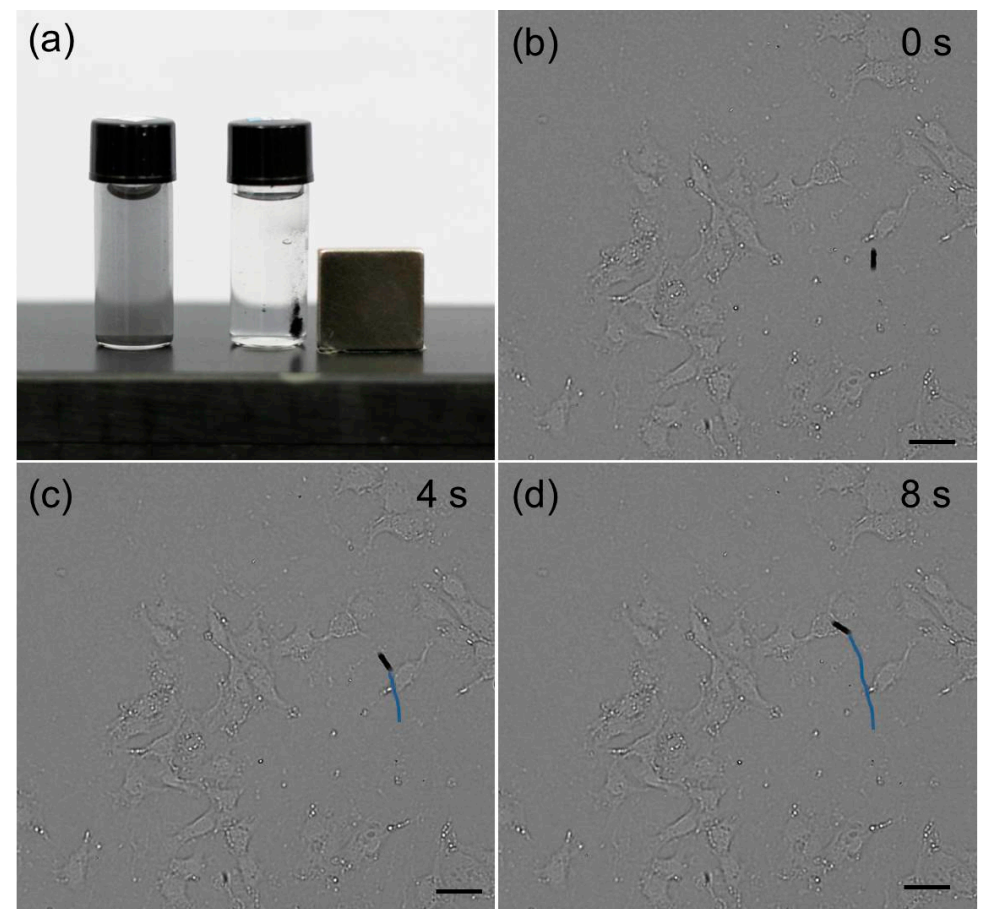

\subsection{Temperature Elevation under Different Concentration of the Micro-Tubes}

Besides the improvement of the magnetic manipulation by integration of $\mathrm{Fe}_{3} \mathrm{O}_{4} \mathrm{NPs}$ to the microtube, more importantly, the assembly of Au NPs on the surface of the microtube allow for the absorption wavelength of the in the NIR region, which resolve the key challenges of Au NPs as 
photothermal agent in cancer therapy. As illustrated in Figure 4a, compare to the maximum wavelength of $\mathrm{Au}$ NPs in the visible region (approximately $520 \mathrm{~nm}$ ), the microtubes assembled $\mathrm{Au}$ and $\mathrm{Fe}_{3} \mathrm{O}_{4}$ NPs shows considerable absortion in NIR region. It is the assembly process causes the aggregation of $\mathrm{Au}$ NPs on the microtubes, leading the maximum adsorption of the microtubes shift to the NIR region. In this way, under the irradiation of the NIR, the Au NPs on the outer surfaces of the $\mathrm{Au}$ NPs-(PGA/SPI $)_{14.5}-\mathrm{Fe}_{3} \mathrm{O}_{4}$ NPs micro-tubes converted the light energy into thermal energy. In addition, the photo-thermal effect increased the temperature of the aqueous dispersion of the $\mathrm{Au}$ NPs-(PGA/SPI $)_{14.5}-\mathrm{Fe}_{3} \mathrm{O}_{4}$ NPs micro-tubes. To investigate the dependence of the elevated temperature on the concentration of the Au NPs-(PGA/SPI $)_{14.5}-\mathrm{Fe}_{3} \mathrm{O}_{4}$ NPs micro-tubes; the suspension, with different concentrations of the Au NPs-(PGA/SPI) $14.5-\mathrm{Fe}_{3} \mathrm{O}_{4}$ NPs micro-tubes, were irradiated by NIR with a wavelength of $808 \mathrm{~nm}$ and a power output of 2 watts. Figure 4 shows the temperature elevation under the exposure of the NIR at $30 \mathrm{~s}$ intervals over $10 \mathrm{~min}$. The sample's temperature was elevated by increasing the period of the NIR. The elevated temperature rose, in the range of 0.05-4 $\mathrm{mg} / \mathrm{mL}$, when caused by the concentration within the micro-tubes. When treated for $10 \mathrm{~min}$ by NIR, the sample's temperature, with a concentration of $0.5 \mathrm{mg} / \mathrm{mL}$, exceeded $42{ }^{\circ} \mathrm{C}$. This temperature was enough to induce apoptosis of the cancer cell [14]. In contrast, no substantial change of temperature was observed when the NIR was exposed to distilled water, which indicated the photo-thermal effect of $\mathrm{Au}$ NPs-(PGA/SPI $)_{14.5}-\mathrm{Fe}_{3} \mathrm{O}_{4}$ NPs, micro-tubes. As a versatile platform of assembling functional components, the microtube also allows for other external physical guidance to the defined location. If needed, the affinity components such as antibody, aptamer and peptides onto the microtube to perform the specific recognition.

Figure 4. Heating effect of the microtube under the near infrared radiation (NIR) irradiation. (a) UV-vis spectrum of the bold microtube (black line), Au NPs (red line), and $\mathrm{Au}$ NPs-(PGA/SPI $)_{14.5}-\mathrm{Fe}_{3} \mathrm{O}_{4}$ NPs micro-tube (blue line); (b) The elevated temperature of suspension of the Au NPs-(PGA/SPI $)_{14.5}-\mathrm{Fe}_{3} \mathrm{O}_{4}$ NPs micro-tubes with different concentration $(0.05,0.1,0.2,0.5,1.0,2.0,4.0 \mathrm{mg} / \mathrm{mL})$ under the irradiation of NIR conducted every $30 \mathrm{~s}$ over $10 \mathrm{~min}$.
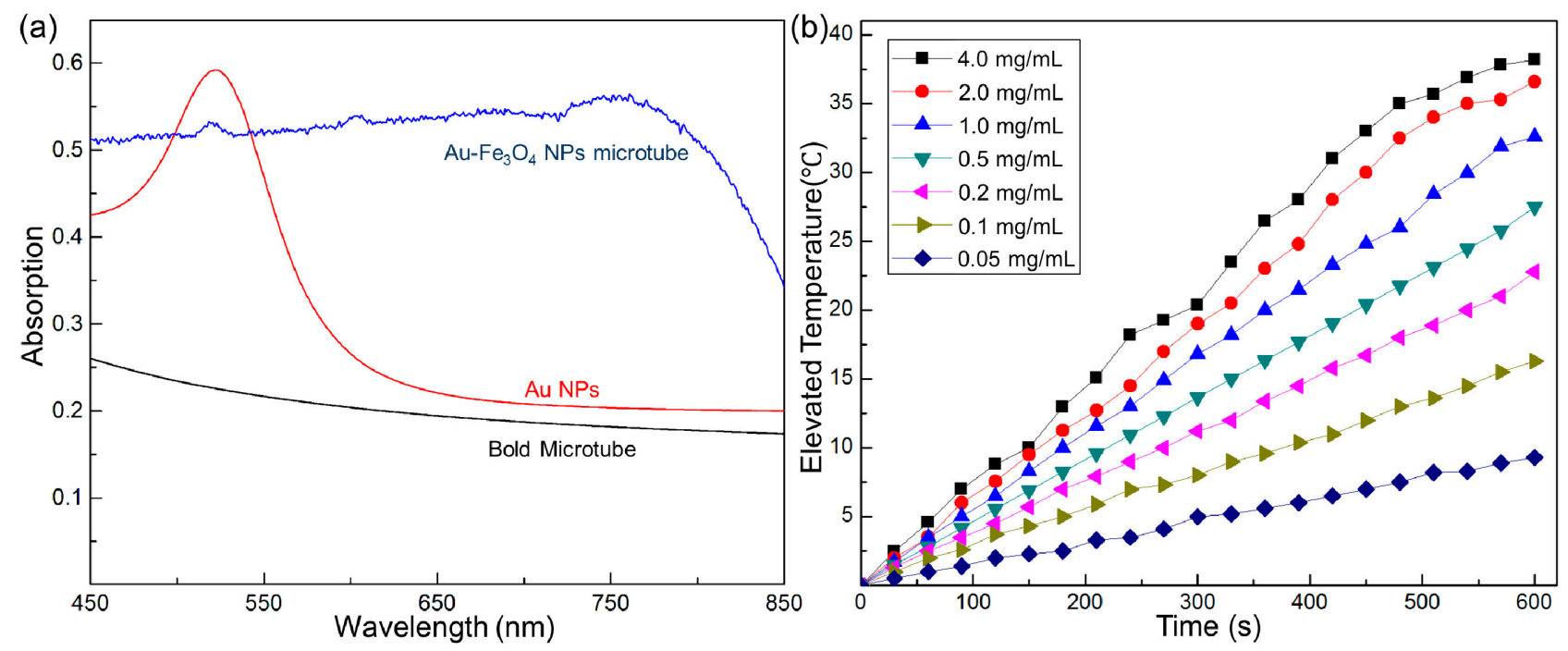


\subsection{Biodegradable Behavior of the Micro-Tubes}

When the micro-tubes were degraded under the treatment of $\alpha$-chymotrypsin, the Au NPs-(PGA/SPI $)_{14.5}-\mathrm{Fe}_{3} \mathrm{O}_{4}$ NPs micro-tubes also showed biodegradable property. The comparison of SEM images of the micro-tubes, before and after the treatment of $\alpha$-chymotrypsin, verified the biodegradability of the micro-tubes (Figure 5). The SEM image of the enzyme-degraded micro-tubes confirmed the building walls of the micro-tubes became thinner in contrast with the micro-tubes before degrading; in addition, the degraded micro-tubes exhibited a rough surface and collapsed geometry. In the control experiment, a micro-tube was stabilized at $4{ }^{\circ} \mathrm{C}$ over one month. This result demonstrates that the assembled $\mathrm{Au}$ NPs-(PGA/SPI $)_{14.5}-\mathrm{Fe}_{3} \mathrm{O}_{4}$ NPs micro-tubes are biodegradable. The biodegradable behavior of each micro-tube was verified because one of the components of the micro-tube, the soybean protein, was, without difficulty, hydrolyzed by the $\alpha$-chymotrypsin. The biodegradable property of the micro-tubes extended the application of the micro-tubes especially in the field of biology and medicine.

Figure 5. Biodegradable behavior of the $\mathrm{Au}$ NPs-(PGA/SPI $)_{14.5}-\mathrm{Fe}_{3} \mathrm{O}_{4}$ NPs micro-tubes. (a) This photograph shows the SEM images of the micro-tubes before the treatment of chymotrypsin; (b) This photograph shows the SEM images of the micro-tubes after the treatment of $\alpha$-chymotrypsin. Scale bar $=10 \mu \mathrm{m}$.

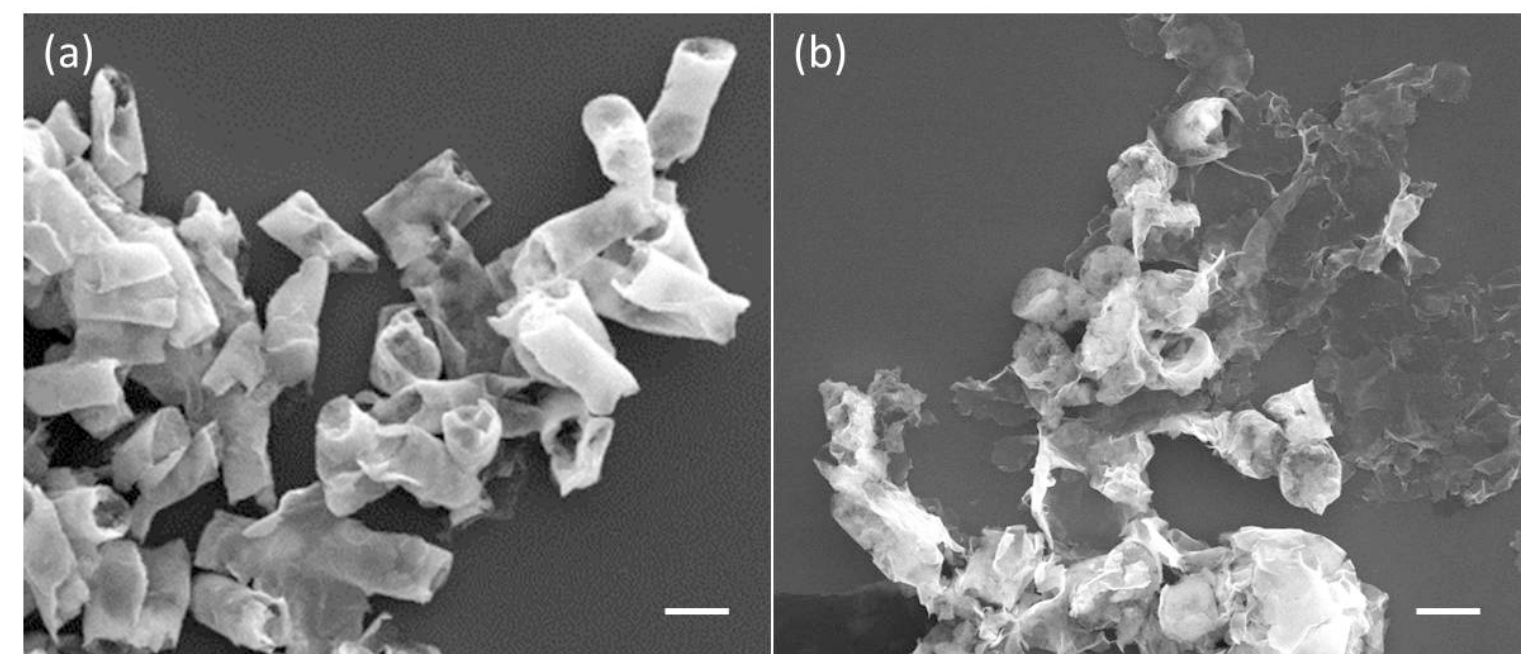

\subsection{The Photothermal Effect of the Micro-Tubes to the Viability of HeLa Cells}

The Au NPs-(PGA/SPI $)_{14.5}-\mathrm{Fe}_{3} \mathrm{O}_{4}$ NPs micro-tubes directed to the HeLa cell, under the irradiation of NIR, induced apoptosis of the HeLa cells through the photothermal effect of the micro-tubes [15]. According to the results in Figure 4a, the photothermal effect of the micro-tubes, when subjected to the radiation of the NIR, heated the cell culture and induced apoptosis of the cells at a temperature far beyond $42{ }^{\circ} \mathrm{C}$. In order to evaluate the photothermal effect of the micro-tubes to the HeLa cells, propidium iodide (PI), a fluorescent cell dye, indicated the apoptosis of the cells by the penetration into the nuclei of the cells through the breakage of membrane of the apoptosis cells. 
The solution of PI was added into the cell culture to confirm the apoptosis of the HeLa cells. The fluorescence image (Figure 6d) was taken when the micro-tube located to the HeLa cells (Figure 6c) after NIR radiation for one minute and PI staining for $10 \mathrm{~min}$. The image exhibits the red fluorescence from the nuclei of the HeLa cells surrounding the micro-tubes. In contrast, no red fluorescence was observed at approximately $50 \mu \mathrm{m}$ from the micro-tube where the photo-thermal effect was negligible. The red fluorescence is from the interaction of PI and nucleic acid. PI can penetrate into the nuclei from the breakage of cell membrane and such method has been widely used to evaluate apoptosis of cells. Figure $6 \mathrm{~d}$ shows the red fluorescence from the nuclei of the cells surrounding the microtube verify distinct apoptotic feature of cells, indicating the photothermal effect of the micro-tubes, under radiation, induced apoptosis of the HeLa cells. When guided by the external magnetic field, the micro-tube induced apoptosis of the HeLa cells near the target location. This outcome, under the combined treatment of magnetic guidance and the irradiation of the NIR, confirmed the target photo-thermal therapy on the cancer cells. Moreover, considered the biodegradable property of the $\mathrm{Au}$ NPs-(PGA/SPI $)_{14.5}-\mathrm{Fe}_{3} \mathrm{O}_{4}$ NPs micro-tubes, the multifunctional micro-tubes will be degraded by enzymes after their photo-thermal performance, suggesting promising potential application in vivo.

Figure 6. The target photothermal effect of the $\mathrm{Au}$ NPs-(PGA/SPI $)_{14.5}-\mathrm{Fe}_{3} \mathrm{O}_{4} \mathrm{NPs}$ micro-tube to the viability of HeLa cells when under NIR treatment. (a-c) Time-lapse images illustrating the target movement of the Au NPs-(PGA/SPI $)_{14.5}-\mathrm{Fe}_{3} \mathrm{O}_{4}$ NPs micro-tube to the HeLa cells directed by the external magnetic field; (d) This photograph shows the corresponding fluorescent image (c) of the micro-tube after the radiation of the NIR. Scale bar $=20 \mu \mathrm{m}$.

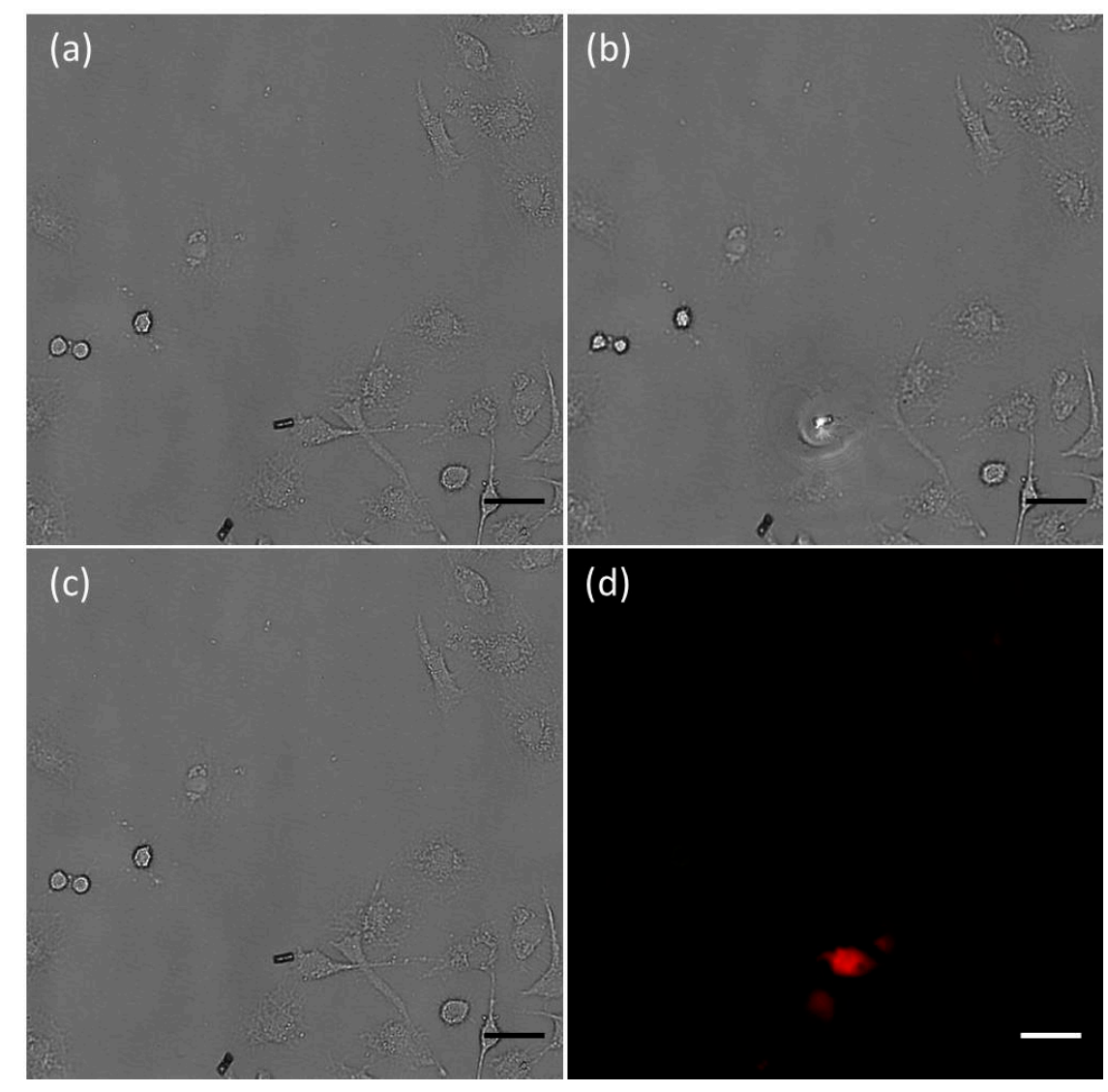




\section{Experimental}

\subsection{Materials}

Commercially available porous polycarbonate (PC) membranes with a pore diameter of $5 \mu \mathrm{m}$ were purchased from the Whatman Corporation-United Kingdom. The Polyethyleneimine $\left(M_{\mathrm{W}}=70,000 \mathrm{Da}\right.$; 50 wt \% solution in water) and the Propidium Iodide (PI) were purchased from Sigma-Aldrich Shanghai Trading Co., Ltd., Shanghai, China. The soybean protein isolate (SPI), containing 86.7\% protein, was purchased from Gushen Biological Technology Group Co., Ltd., Shandong, China. The Pancreatin powder was obtained from Yakanglinuo Bioengineering Co., Ltd., Jinan, China. The $\mathrm{HAuCl}_{4} \cdot 4 \mathrm{H}_{2} \mathrm{O}, \mathrm{FeCl}_{2} \cdot 4 \mathrm{H}_{2} \mathrm{O}, \mathrm{FeCl}_{3} \cdot 4 \mathrm{H}_{2} \mathrm{O}$, citric acid monohydrate, $\mathrm{NaCl}, \mathrm{CH}_{2} \mathrm{Cl}_{2}$, and ethanol were used without further purification. The water used in all experiments was prepared in a Milli-Q purification system with the resistivity higher than $18.2 \mathrm{M} \Omega \cdot \mathrm{cm}^{-1}$.

\subsection{Preparation of Citrate-Stabilized $\mathrm{Fe}_{3} \mathrm{O}_{4}$ Nanoparticles}

The citrate stabilized $\mathrm{Fe}_{3} \mathrm{O}_{4}$ NPs were prepared according to the method of Sahoo et al. [16]. Briefly, $1.40 \mathrm{~g} \mathrm{FeCl}_{3} \cdot 4 \mathrm{H}_{2} \mathrm{O}$ and $0.86 \mathrm{~g} \mathrm{FeCl}_{2} \cdot 4 \mathrm{H}_{2} \mathrm{O}$ were dissolved in $50 \mathrm{~mL}$ of distilled water degassed by nitrogen gas. The solution was vigorously stirred by the Stirring Hotplates (Thermal Scientific, Waltham, MA, USA) and heated, under nitrogen protection, for $30 \mathrm{~min}$ to $80^{\circ} \mathrm{C}$; then, $8 \mathrm{~mL}$ of $\mathrm{NH}_{4} \mathrm{OH}$ was added to the solution, this solution then turned black and was heated for another $30 \mathrm{~min}$. The $\mathrm{Fe}_{3} \mathrm{O}_{4}$ nanoparticles were collected by a magnet and were then rinsed three times. The citric acid solution $(4 \mathrm{~mL}, 0.24 \mathrm{~g} / \mathrm{mL})$ was then added to the solution, and the solution was then heated to $95{ }^{\circ} \mathrm{C}$, once again under nitrogen protection. The reaction solution was then cooled to room temperature, while still under nitrogen protection. The $\mathrm{Fe}_{3} \mathrm{O}_{4}$ nanoparticles were collected by a magnet and were rinsed three times using distilled water. The $\mathrm{Fe}_{3} \mathrm{O}_{4}$ nanoparticles suspension was degassed by nitrogen and kept at $4{ }^{\circ} \mathrm{C}$.

\subsection{Preparation of Citrate Coated Au Nanoparticles}

To prepare the citrus-coated Au-nanoparticles a solution of $0.5 \mathrm{~mL}$ of $4.0 \times 10^{-2} \mathrm{M} \mathrm{HAuCl}_{4}$ was added to $100 \mathrm{~mL}$ of distilled water, followed by the addition of $1 \mathrm{~mL}$ of $5.0 \times 10^{-2} \mathrm{M}$ Trisodium citrate. After waiting five $\min , 0.6 \mathrm{~mL}$ of ice cold $0.1 \mathrm{M} \mathrm{NaBH}_{4}$ was added to this solution, this solution was then vigorously stirred for $10 \mathrm{~min}$ by the Stirring Hotplates. The color of the solution gradually turned red to indicate the formation of the gold nanoparticles. The Au NPs solution was kept at $4{ }^{\circ} \mathrm{C}$ before experimentation [17].

\subsection{Preparation of Soybean Protein Isolate Micro-Tube}

The framework of the soybean protein isolate micro-tubes was prepared through the integration of the PGA and the SPI to the PC membrane [18]. Prior to use, the PGA solution ( $1 \mathrm{mg} / \mathrm{mL}$ in $0.5 \mathrm{M} \mathrm{NaCl})$, and the soybean protein isolated solution $(1 \mathrm{mg} / \mathrm{mL}$ in PBS solution with $\mathrm{pH}$ of 7.2) together with the $\mathrm{Fe}_{3} \mathrm{O}_{4}$ NPs solution were filtered with a $0.45 \mu \mathrm{m}$ filter. The PC membrane in conjunction with the template was alternatively immersed into the positive charged PGA solution and the negative charged 
soybean protein solution for one hour as one bilayer. The immediate rinsing step, before immersing into the polyelectrolyte solution, was performed three times. When the 14.5 bilayers of soybean protein were isolated and the PGA was deposited into the template, the template was then immersed into the $\mathrm{Fe}_{3} \mathrm{O}_{4}$ NPs solution for one hour. These components adsorbed both surfaces of the template and were later removed by using wet cotton swabs. The micro-tubes were obtained by dissolving the templates with $\mathrm{CH}_{2} \mathrm{Cl}_{2}$. The micro-tubes were then collected by external magnetic field and rinsed in ethanol and water.

The assembly of Au NPs to the outer surfaces of the soybean protein isolate micro-tubes was performed by incubating the micro-tubes with citric-stabilized Au NPs for one hour at an approximate room temperature of $27^{\circ} \mathrm{C}$. The micro-tubes were separated using an external magnetic field. The micro-tubes, in solution, performed and then directed movement to the external magnetic field. The movement behavior was recorded by microscope.

\subsection{Characterization of the Resulting Micro-Tube}

A Hitachi S-4300 instrument using an operating voltage of $10 \mathrm{keV}$ conducted the SEM and EDX analysis. For an examination of the micro-tubes, a droplet of each of the micro-tube's solution was trickled on to a silicon wafer. The TEM images were conducted by using a Tecnai G2 F30 microscope operated at $120 \mathrm{kV}$. The micro-tube samples were prepared on a carbon-coated copper grid. An Olympus BX53F optical microscope recorded the movement of the micro-tubes and the photothermal effect on the HeLa cells.

\subsection{Cell Culture}

The HeLa cells were incubated in a DMEM medium containing $10 \%$ fetal calf serum and $1 \%$ penicillin and streptomycin at 5\% $\mathrm{CO}_{2}$ : with humidity. The HeLa cells were divided into a $60 \mathrm{~mm}$ polystyrene Petri dish using a standard trypsin-based technique. After the cells were cultivated in the logarithmic growth phase, the micro-tubes were added to the cell culture [19].

\subsection{Degradable Behavior of the Micro-Tube}

The micro-tubes were exposed to $\alpha$-chymotrypsin solution $(5 \mathrm{U} / \mathrm{mL})$ in a phosphate buffer solution (PBS) with a $\mathrm{pH} 7.4$ at room temperature overnight. In the control experiment, the $\mathrm{Au}$ NPs-(PGA/SPI) ${ }_{14.5}-\mathrm{Fe}_{3} \mathrm{O}_{4}$ NPs micro-tubes were incubated in a plain PBS with a $\mathrm{pH}$ of 7.4 at a room temperature of approximately $27^{\circ} \mathrm{C}$. For SEM characterization, the samples were removed from the incubation solution and washed three times.

\subsection{Analysis of the Photo-Thermal Effort of Micro-Tube under the Irradiation of NIR}

The cell culture containing the micro-tubes were irradiated for one minute by using a continuous-wave diode NIR laser (Wuhan Huaray Precision Laser Co., Ltd., Wuhan, China) with a center wavelength of $808 \pm 10 \mathrm{~nm}$ and output power of 1-5 W. Following the irradiation of the NIR, the viability of the HeLa cells was assessed by performing an apoptosis assay by adding $5 \mu \mathrm{L}$ of the PI solution to the cell culture. After staining the HeLa cells for 20 min by using Propidium iodide (PI), the fluorescent image 
of the HeLa cell was taken by an Olympus BX53F optical microscope with an objective of $20 \times$ and was then used to confirm the apoptosis of the HeLa cells [20].

\section{Conclusions}

This experiment has described a $\mathrm{Fe}_{3} \mathrm{O}_{4}$ NPs and $\mathrm{Au}$ NPs functionalized biodegradable micro-tube based on the integration of polymers to a porous template. The size and geometry of each micro-tube was dependent on the template. The locations of the magnetic micro-tubes were controlled by applying an external magnetic field. Of interest, when under-exposed to the NIR, the Au NPs, on the outer surfaces of the micro-tubes converted the light energy to a heat source, this photo-thermal effect elevated the temperature of the aqueous suspension beyond $42{ }^{\circ} \mathrm{C}$. In this instance, and when under the radiation of the NIR, the micro-tubes located near the cancer cells were able to induce the apoptosis of the HeLa cells. Furthermore, due to the composition of the construct of the micro-tubes, the SPI enable the micro-tubes to degrade by the enzymatic treatment. As the similar photothermal agent was applied in vivo [21], the biodegradable and multifunctional micro-tubes have great potential for remotely controlling photothermal therapy, and more deep studies in vivo are being conducted.

\section{Acknowledgments}

This work was supported by the grants from the National Natural Science Foundation of China (No. 31071493).

\section{Author Contributions}

We thank Natural Science Foundation, R.O.C. for financial support of this research.

\section{Conflicts of Interest}

The authors declare no conflict of interest.

\section{References}

1. Sharman, W.M.; Allen, C.M.; van Lier, J.E. Photodynamic therapeutics: Basic principles and clinical applications. Drug Discov. Today 1999, 4, 507-517.

2. Dolmans, D.E.J.G.J.; Fukumura, D.; Jain, R.K. Photodynamic therapy for cancer. Nat. Rev. Cancer 2003, 3, 380-387.

3. Zharov, V.P.; Mercer, K.E.; Galitovskaya, E.N.; Smeltzer, M.S. Photothermal nanotherapeutics and nanodiagnostics for selective killing of bacteria targeted with gold nanoparticles. Biophys. J. 2006, 90, 619-627.

4. Li, J.; Gu, M. Surface plasmonic gold nanorods for enhanced two-photon microscopic imaging and apoptosis induction of cancer cells. Biomaterials 2010, 31, 9492-9498.

5. Delcea, M.; Sternberg, N.; Yashchenok, A.M.; Georgieva, R.; Bäumler, H.; Möhwald, H.; Skirtach, A.G. Nanoplasmonics for dual-molecule release through nanopores in the membrane of red blood cells. ACS Nano 2012, 6, 4169-4180. 
6. Liang, X.; Li, X.; Yue, X.; Dai, Z. Conjugation of porphyrin to nanohybrid cerasomes for photodynamic diagnosis and therapy of cancer. Angew. Chem. Int. Ed. 2011, 50, 11622-11627.

7. Ke, H.; Wang, J.; Dai, Z.; Jin, Y.; Qu, E.; Xing, Z.; Guo, C.; Yue, X.; Liu, J. Gold-nanoshelled microcapsules: A theranostic agent for ultrasound contrast imaging and photothermal therapy. Angew. Chem. Int. Ed. 2011, 50, 3017-3021.

8. Son, S.J.; Reichel, J.; He, B.; Schuchman, M.; Lee, S.B. Magnetic nanotubes for magnetic-fieldassisted bioseparation, biointeraction, and drug delivery. J. Am. Chem. Soc. 2005, 127, 7316-7317.

9. Köhler, K.; Möhwald, H.; Sukhorukov, G.B. Thermal behavior of polyelectrolyte multilayer microcapsules: 2. Insight into molecular mechanisms for the PDADMAC/PSS system. J. Phys. Chem. 2006, 110, 24002-24010.

10. Wang, F.; Liu, P.; Nie, T.; Wei, H.; Cui, Z. Characterization of a polyamine microsphere and its adsorption for protein. Int. J. Mol. Sci. 2013, 14, 17-29.

11. Lee, D.; Cohen, R.E.; Rubner, M.F. Heterostructured magnetic nanotubes. Langmuir 2007, 23, 123-129.

12. Sahoo, Y.; Goodarzi, A.; Swihart, M.T.; Ohulchanskyy, T.Y.; Kaur, N.; Furlani, E.P.; Prasad, P.N. Aqueous ferrofluid of magnetite nanoparticles: Fluorescence labeling and magnetophoretic control. J. Phys. Chem. B 2005, 109, 3879-3885.

13. Wu, Z.; Lin, X.; Wu, Y.; Si, T.; Sun, J.; He, Q. Near-infrared light-triggered “on/off” motion of polymer multilayer rockets. ACS Nano 2014, 8, 6097-6105.

14. Mazumder, A.; Shivashankar, G.V Gold-nanoparticle-assisted laser perturbation of chromatin assembly reveals unusual aspects of nuclear architecture within living cells. Biophys. J. 2007, 93, 2209-2216.

15. Steinkamp, J.A.; Lehnert, B.E.; Lehnert, N.M. Discrimination of damage/dead cells by propidium iodide uptake in immunofluorescently labeled populations analyzed by phase-sensitive flow cytometry. J. Immunol. Methods 1999, 226, 59-70.

16. Grabar, K.C.; Allison, K.J.; Baker, B.E.; Bright, R.M.; Brown, K.R.; Freeman, R.G.; Fox, A.P.; Keating, C.D.; Musick, M.D.; Natan, M.J. Two-Dimensional arrays of colloidal gold particles: A flexible approach to macroscopic metal surfaces. Langmuir 1996, 12, 2353-2361.

17. Brown, K.R.; Natan, M.J. Hydroxylamine seeding of colloidal au nanoparticles in solution and on surfaces. Langmuir 1998, 14, 726-728.

18. Roy, C.J.; Jonas, A.M.; Landoulsi, J. Growth mechanism of confined polyelectrolyte multilayers in nanoporous templates. Langmuir 2010, 2, 3350-3355.

19. Sioud, M.; Mobergslien, A. Selective killing of cancer cells by peptide-targeted delivery of an anti-microbial peptide. Biochem. Pharmacol. 2012, 84, 1123-1132.

20. Murayama, H.; Sadakane, K.; Yamanoha, B.; Kogure, S. Low-power $808 \mathrm{~nm}$ laser irradiation inhibits cell proliferation. Lasers Med. Sci. 2012, 27, 87-93.

21. Zhang, Z.; Wang, J. ; Nie, X.; Wen, T.; Ji, Y.; Wu, X.; Zhao, Y. Near infrared laser-induced targeted cancer therapy using thermoresponsive polymer encapsulated gold nanorods. J. Am. Chem. Soc. 2014, 136, 7317-7326.

(C) 2014 by the authors; licensee MDPI, Basel, Switzerland. This article is an open access article distributed under the terms and conditions of the Creative Commons Attribution license (http://creativecommons.org/licenses/by/3.0/). 